\title{
Relacionamento conjugal e características sociodemográficas de casais heteroafetivos
}

\section{Marital relationship and sociodemographic characteristics of hetero-affective couples}

\section{Relación conyugal y características sociodemográficas de parejas heterosexuales}

\section{Beatriz Schmidt*}

Universidade Federal do Rio Grande do Sul - UFRGS, Porto Alegre, Rio Grande do Sul, Brasil

\author{
Simone Dill Azeredo Bolze** \\ Universidade Federal de Santa Catarina - UFSC, Florianópolis, Santa Catarina, Brasil
}

Mauro Luis Vieira***

Universidade Federal de Santa Catarina - UFSC, Florianópolis, Santa Catarina, Brasil

\section{Maria Aparecida Crepaldi**}

Universidade Federal de Santa Catarina - UFSC, Florianópolis, Santa Catarina, Brasil

\begin{abstract}
RESUMO
O presente estudo objetivou descrever a relação entre relacionamento conjugal e características sociodemográficas em arranjos familiares heteroafetivos. Participaram da pesquisa 208 adultos (104 mulheres e 104 homens), com média de 30 a 40 anos de idade e 10 anos de relação conjugal. Os instrumentos utilizados foram o Questionário Sociodemográfico e o Questionário Floreal. Os dados da pesquisa foram submetidos ao Statistical Package for Social Sciences e analisados de forma descritiva e inferencial. A relação de casal dos respondentes se caracterizou, em média, por harmonia conjugal. Identificou-se que quanto menor a renda e menor a escolaridade, mais os cônjuges referiram interações permeadas por reciprocidade negativa e evitação. No mesmo sentido, o número de pessoas residentes no domicílio familiar também se apresentou positivamente relacionado à emergência de brigas, discussões, trocas de acusações e sentimentos de desigualdade entre o casal, bem como à retração feminina com o intento de evitar conflitos conjugais. As mulheres pareceram mais sensíveis às relações entre conjugalidade e variáveis sociodemográficas em comparação aos homens.
\end{abstract}

Palavras-chave: relações conjugais, relações homem-mulher, demografia.

\section{ABSTRACT}

The present study aimed to describe the relationship between marital relationship and sociodemographic characteristics in hetero-affective family 
arrangements. Participated in the survey 208 adults (104 women and 104 men), with average from 30 to 40 years old and 10 years of marital relationship. The instruments used were the Sociodemographic Questionnaire and the Floreal Questionnaire. The survey data were submitted to the Statistical Package for Social Sciences and analyzed descriptively and inferentially. The marital relation of the respondents was characterized, on average, by marital harmony. It was found that the lower the income and the education were, more the spouses reported interactions permeated by negative reciprocity and avoidance. Similarly, the amount of people living in the family home also performed positively related to the emergence of fights, arguments, exchanges of accusations and feelings of inequality between the couple and the female retraction with intent to avoid marital conflicts. Women appeared to be more sensitive to the relationship between marital affairs and socio-demographic variables when compared to men.

Keywords: marital relationships, man-woman relationship, demography.

\section{RESUMEN}

Este estudio tuvo como objetivo describir la relación entre la relación conyugal y características socio demográficas de los arreglos heteroafectivos familiares. Participaron de la investigación 208 adultos (104 mujeres y 104 hombres), con media de 30 a 40 años de edad y 10 años de relación conyugal. Los instrumentos utilizados fueron el Cuestionario Sociodemográfico y el Cuestionario Floreal. Los datos de la encuesta fueron introducidos en el programa Statistical Package for Social Sciences y analizados de forma descriptiva y por inferencia. La relación de pareja de los encuestados se caracterizó, en promedio, por la armonía conyugal. Se identificó que cuanto menor la renta y menor la escolaridad, más los conyugues refirieron interacciones permeadas por reciprocidad negativa y evitación. En el mismo sentido, la cantidad de personas residentes en el domicilio familiar también se presentó positivamente relacionada a la aparición de peleas, discusiones, intercambios de acusaciones y sentimientos de desigualdad entre la pareja, así como a la retracción femenina con el intento de evitar conflictos conyugales. Las mujeres parecieron más sensibles a las relaciones entre conyugalidad y variables socio demográficas en comparación a los hombres.

Palabras-clave: relaciones conyugales, relaciones hombre-mujer, demografía.

\section{I ntrodução}

Uma das tarefas mais difíceis e complexas do ciclo de vida familiar é tornar-se casal (Carter \& McGoldrick, 1995; Heckler \& Mosmann, 2014; Rolim \& Wendling, 2013). O processo de formação da conjugalidade compreende diferentes contextos e níveis da relação, 0 que deriva na acepção psicossocial de um relacionamento afetivo estável (Féres-Carneiro \& Diniz Neto, 2010). O casamento, definido como um modelo adulto de intimidade (Bolze, Crepaldi, Schmidt, \& Vieira, 2013; Whitaker, 1995), exige que duas pessoas que possuem vidas pregressas distintas, encontrem-se e renegociem uma série de questões já anteriormente definidas para si de modo individual, ou 
por suas famílias de origem, envolvendo, por exemplo, como e quando comer, trabalhar, dormir, recrear-se, conversar, discutir e fazer sexo (Carter \& McGoldrick, 1995; Schmidt, 2012). Tais reordenamentos nas vivências individuais ocorrem no sentido de buscar construir uma história de vida compartilhada e uma identidade conjugal (Féres-Carneiro \& Diniz Neto, 2010). Além dessas questões, são definidas ainda as relações com as famílias de origem, amizades e vida social. De tal forma, o casamento não se restringe exclusivamente aos vínculos interpessoais estabelecidos por duas pessoas - ele diz respeito ainda a um contexto ecossistêmico mais amplo, no qual o casal está inserido e interage (Willi, 1995).

O contexto de inserção dos cônjuges tem uma importante repercussão no modo de organização do casal e da família (Vieira, Lacerda, Vieira, \& Seidl-de-Moura, 2011). Assim, ressalta-se que a maneira de pensar o casamento e a vida familiar sofreu importantes modificações a partir das transformações socioeconômicas e culturais desencadeadas em meados do século $X X$, notadamente após a II Guerra Mundial (Coutinho \& Menandro, 2010). Dentre os fatores que se associam às novas demandas e à redefinição do casamento contemporaneamente, destacam-se: maior liberdade sexual, emancipação da mulher, duplo ingresso dos cônjuges no mercado de trabalho, possibilidade de separação conjugal ou de divórcio e, adicionalmente, características individualistas cada vez mais fortes em nossa cultura (Heckler \& Mosmann, 2014).

Dado o processo de transformação pelo qual o casamento vem passando nas últimas décadas, discute-se que os laços conjugais contemporâneos necessitam compreender um espaço em que as forças da conjugalidade e da individualidade de cada membro da díade coexistam e interajam (Féres-Carneiro, 1998; Féres-Carneiro \& Diniz Neto, 2010; Scorsolini-Comin \& Santos, 2011a). Assim, as relações conjugais se constituem a partir de identidades particulares de cada cônjuge e se mantêm à medida que contribuem para a promoção de crescimento pessoal (Féres-Carneiro, Ponciano, \& Magalhães, 2007). Desse modo, além de almejarem casar e/ou recasar (Zordan, Wagner, \& Mosmann, 2012), as pessoas estão também motivadas a constituir uma relação conjugal de qualidade, mutuamente satisfatória para ambos os cônjuges (Perlin, 2006).

A satisfação conjugal se refere a um fenômeno complexo, que pode ser definido como a avaliação pessoal de cada um dos cônjuges sobre a qualidade do relacionamento de casal (Narciso \& Ribeiro, 2009). A capacidade dos membros da díade de se ajustar à vida conjugal em um dado momento é o que define a qualidade conjugal (Askari, Noah, Hassan, \& Baba, 2012). Dentre os elementos que podem ser levados em conta no processo de avaliação subjetiva da qualidade do relacionamento de casal, constam frequência e intensidade de 
manifestações comportamentais de reciprocidade negativa, evitação e harmonia conjugal (Bolze et al., 2013).

Casais que se consideram infelizes parecem ser mais negativos emocionalmente, bem como mais propensos a serem reciprocamente negativos nas interações diádicas em comparação a casais que se consideram felizes (Féres-Carneiro \& Diniz Neto, 2010). Essas diferenças são constatadas inclusive em situações de conflito conjugal, em que casais infelizes tendem a demonstrar menos afeto positivo, concordância e aprovação, além de mais críticas ao cônjuge do que casais felizes (Madhyastha, Hamaker, \& Gottman, 2011). Ademais, a evitação se refere à esquiva de conversas que exijam a exposição de sentimentos e, eventualmente, de fraquezas pessoais, com o intuito de que os problemas se resolvam ao longo do tempo (Bolze et al., 2013; Schmidt, 2012). Os comportamentos evitativos são considerados disfuncionais, associando-se ao maior risco de insatisfação conjugal, pois os descontentamentos com a relação de casal são menos manifestados, mas não menos sentidos (Colossi \& Falcke, 2013).

A harmonia conjugal, por sua vez, pode ser definida por um sentimento de empatia, o qual favorece que cada um dos membros do casal perceba-se acolhido, validado em seus sentimentos e respeitado na relação com o parceiro (Oliveira, Falcone, \& Ribas, 2009). Desse modo, um casal pode ser considerado harmônico se esse se percebe como feliz e ajustado, além de demonstrar um alto nível de concordância nos vários âmbitos que concernem sua vida em comum (Silvares \& Souza, 2008). Além disso, a expressão do afeto nas discussões e na ocorrência de discordâncias de ideias, com expressão de preocupação, cuidado e respeito mútuo, parece ser uma forma mais positiva de lidar com os conflitos (Bolze et al., 2013).

Posto isso, adiciona-se que múltiplos fatores se associam ao processo da percepção da satisfação e da qualidade na conjugalidade, dentre os quais estão experiências que cada cônjuge traz de suas famílias de origem (Wagner \& Falcke, 2001), características de personalidade dos membros de casal, forma como eles constroem a relação amorosa, além de variáveis sociodemográficas, como sexo, grau de escolaridade, nível cultural, nível socioeconômico, trabalho remunerado, número e presença de filhos em casa (Norgren, Souza, Kaslow, Hammerschmidt, \& Sharlin, 2004; Scorsolini-Comin \& Santos, 2011b).

No presente estudo, parte-se do pressuposto de que a satisfação e a qualidade conjugal são influenciadas, dentre outros fatores, pelas variáveis do contexto social e demográfico no qual o casal está inserido. A literatura aponta que casais com níveis de escolaridade mais altos tendem a apresentar maior satisfação conjugal (Conger, Conger, \& Martin, 2010). Outrossim, há indicativos de que a satisfação conjugal declina por conta do número de filhos (Wendorf, 
Lucas, Imamoğlu, Weisfeld, \& Weisfeld, 2011). Não obstante, o contexto parece influenciar, ainda, as táticas de resolução de conflitos adotadas pelos cônjuges, associando-se ao risco para a dissolução conjugal. Características sociodemográficas, tais quais menores níveis de escolaridade e de renda, estão relacionadas a taxas mais elevadas de divórcio (Birditt, Brown, Orbuch, \& McIlvane, 2010).

O baixo nível socioeconômico, especificamente, vincula-se ao aumento da hostilidade entre o casal (Dakin \& Wampler, 2008), bem como à ocorrência e à severidade dos casos de interações conjugais conflituosas. Nesse sentido, é importante destacar que interações conflitivas estão presentes em todas as relações interpessoais, incluindo as conjugais (Schmidt, 2012). Entretanto, hipotetiza-se que a vulnerabilidade socioeconômica pode se constituir em um fator de risco ao desenvolvimento de famílias e de casais, podendo eventualmente fragilizá-los.

Desse modo, em face dos indicativos de que o contexto influencia as interações dos membros da família, destacando-se dentre elas as interações dos cônjuges, o estudo das relações entre conjugalidade e variáveis sociodemográficas apresenta relevância. Isso ocorre, em primeiro lugar, à medida que contribui para o processo de produção de conhecimentos úteis à elaboração de estratégias de intervenção junto a casais, sopesando que a qualidade da relação conjugal se constitui em fator de proteção ao desenvolvimento individual e familiar: pessoas que estabelecem interações conjugais satisfatórias são mais longevas, fisicamente saudáveis e desempenham mais adequadamente suas funções parentais (Rauer, Karney, \& Gavan, 2008). Em segundo lugar, traz maior visibilidade ao fenômeno, permitindo ampliar os conhecimentos referentes ao modo como essas relações influenciam as trajetórias desenvolvimentais de indivíduos, casais e famílias. Em terceiro lugar, dadas as particularidades do casamento contemporâneo, as quais o tornam diferente do modelo tradicional e impactam a dinâmica da relação de casal, evidencia-se a necessidade de novos estudos sobre a temática, sobretudo considerando especificidades do contexto brasileiro (Heckler \& Mosmann, 2014), vez que os delineamentos das uniões conjugais vêm se transformando ao longo dos anos (Scorsolini-Comin \& Santos, 2011b).

Assim, o objetivo do presente estudo é descrever a relação entre relacionamento conjugal e características sociodemográficas em arranjos familiares heteroafetivos. De modo específico, pretende-se comparar e correlacionar características do relacionamento conjugal (designadamente harmonia conjugal, reciprocidade negativa e evitação) com características sociodemográficas (notadamente escolaridade, renda, número de pessoas que reside no domicílio familiar, idade dos cônjuges e tempo de união conjugal). Dados os resultados obtidos em estudos internacionais sobre a temática (Birditt 
et al., 2010; Conger et al., 2010; Dakin \& Wampler, 2008; Wendorf et al., 2011), a expectativa é que nacionalmente as características da relação conjugal também variem em função das características sociodemográficas.

\section{Método}

\subsection{Participantes}

O estudo foi realizado junto a 104 casais heteroafetivos residentes em quatro cidades de um Estado do sul do Brasil, perfazendo o total de 208 participantes. Foram incluídos casais cuja união ocorreu de maneira formal (por meio do casamento civil e/ou religioso) ou informal (mediante o que se denomina juridicamente no Brasil por "união estável"), e que tivessem pelo menos um(a) filho(a), biológico(a) ou não, em idade pré-escolar. Para serem incluídos na amostra, os casais deveriam estar morando juntos, ou seja, residindo no mesmo domicílio, por um período mínimo de seis meses.

A média de idade das mulheres foi 33 anos $(D P=6,28)$ e dos homens 36 anos $(D P=7,38)$. O tempo médio de união conjugal foi de 10 anos $(D P=5,45)$. A maioria dos casais participantes $(85,6 \%)$ constituía família original ou intacta, isto é, família nuclear composta por pais biológicos de todos os filhos. Na maioria dos participantes $(95,1 \%)$, identificou-se que residiam no domicílio familiar exclusivamente os cônjuges e filho(a)(s), ou seja, com ausência de outros parentes adultos ou agregados (família ampliada).

\subsection{Instrumentos}

a) Questionário Sociodemográfico: composto por 15 questões concernentes a variáveis como composição familiar, número de pessoas residentes no domicílio, características da habitação, presença de funcionário contratado para auxiliar nos cuidados com a casa e com a(s) criança(s) (empregada doméstica ou babá, por exemplo), bem como idade, escolaridade, profissão, jornada de trabalho e renda mensal dos membros da família. Tal instrumento foi aplicado exclusivamente às mulheres participantes.

b) Questionário Floreal: elaborado por Bigras (2010), tem o objetivo de avaliar a relação conjugal. Considerando os objetivos propostos no presente estudo, serão analisados os resultados atinentes às seguintes dimensões do instrumento: harmonia conjugal (oito questões relacionadas à exposição verbal de sentimentos, realização de concessões com o objetivo de resolver conflitos, percepção de felicidade e de satisfação na relação), reciprocidade negativa (nove 
questões que versam sobre a ocorrência de brigas em escalada, isto é, nas quais as acusações e as ofensas evoluem em gravidade no decorrer da interação conflitiva, além da sensação de sentimentos de injustiça e de desigualdade na relação) e evitação (oito questões concernentes a comportamentos de retração com o objetivo de evitar desavenças e conversas que exijam a exposição de sentimentos e de fraquezas pessoais, deixando que os problemas conjugais se resolvam com o passar do tempo). As dimensões da relação de casal foram avaliadas por intermédio de uma escala de pontos, variando de 1 (discordo totalmente) a 6 (concordo totalmente). Esse questionário foi aplicado às mulheres e aos homens participantes.

\subsection{Procedimentos de coleta de dados}

A amostra foi constituída por conveniência, a partir do envio de cartas convite aos cônjuges, as quais foram remetidas por intermédio das instituições de educação infantil (a saber, creches ou pré-escolas) frequentadas pelo(a)(s) filho(a)(s) do casal. Após o retorno das cartas convite preenchidas com dados para contato com a díade conjugal, realizou-se o agendamento da visita domiciliar, situação em que ocorreu a aplicação dos instrumentos. Os questionários foram respondidos em cômodos separados da residência familiar, visando que as respostas de um cônjuge não interferissem nas respostas do outro. Houve a assinatura do Termo de Consentimento Livre e Esclarecido (TCLE). A pesquisa foi aprovada pelo Comitê de Ética em Pesquisa com Seres Humanos de uma Universidade Federal situada no sul do Brasil, sob o Certificado no 520/2009.

\subsection{Análise dos dados}

Os dados obtidos foram tabulados e submetidos a análises estatísticas mediante a utilização do Statistical Package for Social Sciences (SPSS) - versão 18.0. Realizou-se, inicialmente, análise quantitativa com base na estatística descritiva, que visou descrever os valores para cada variável, por meio da distribuição de frequências e de porcentagens para as variáveis categóricas, bem como média e desvio-padrão para as variáveis contínuas. Assim, foram descritas as variáveis concernentes às características sociodemográficas e ao relacionamento conjugal dos respondentes.

Na sequência, aplicou-se o teste estatístico de Kolmogorov-Smirnov, com o objetivo de verificar se a distribuição dos escores poderia ser considerada normal. A partir desse momento, os dados receberam tratamento estatístico não-paramétrico, uma vez que os escores das variáveis não se distribuíram de forma normal. Desse modo, foram realizadas as seguintes análises estatísticas: comparação entre grupos (aplicação do teste Mann-Whitney para comparar mulheres e 
homens no que concerne à escolaridade em termos de anos concluídos e à renda mensal, bem como escores femininos e masculinos para harmonia conjugal, reciprocidade negativa e evitação na relação de casal); comparação entre categorias (aplicação do teste qui-quadrado para comparar mulheres e homens no que tange aos níveis de escolaridade e à jornada de trabalho em termos de carga horária semanal); e correlação ( $\rho$ de Spearman) entre os escores das variáveis sociodemográficas e de relacionamento conjugal dos participantes.

\section{Resultados}

\subsection{Descrição das características sociodemográficas dos casais participantes}

A Tabela 1 apresenta as características dos casais participantes no que se refere à composição familiar, ao número de filhos e à renda familiar mensal (isto é, renda dos cônjuges somada a outros proventos possivelmente auferidos por demais membros da família). 


\section{Tabela 1}

Caracteristicas dos casais participantes no que se refere à composição familiar, número de filhose renda familiar mensal

\begin{tabular}{|c|c|}
\hline Características sociodemográficas & Porcentagem (\%) \\
\hline \multicolumn{2}{|l|}{ Composição familiar } \\
\hline Familia nuclear com pai e mãe biológicos de todos os filhos & $85,6 \%$ \\
\hline Familias recasadas com pai e mãe biológicos da criança focal & $6,7 \%$ \\
\hline Familias recasadas com mãe biológica da criança focal & $3,8 \%$ \\
\hline Familias nucleares com pais adotivos da criança focal & $1,9 \%$ \\
\hline Familia recasada com pai biológico da criança focal & $1 \%$ \\
\hline Familia recasada com mãe adotiva da criança focal e padrasto & $1 \%$ \\
\hline \multicolumn{2}{|l|}{ Número de filhos } \\
\hline Familias com 1 filho & $38,5 \%$ \\
\hline Familias com 2 filhos & $42,3 \%$ \\
\hline Familias com 3 filhos & $13,4 \%$ \\
\hline Familias com 4 filhos & $4,8 \%$ \\
\hline Familias com 5 filhos & $1 \%$ \\
\hline \multicolumn{2}{|l|}{ Renda da família em faixas ${ }^{a}$} \\
\hline $\mathrm{R} \$ 601,00 \mathrm{a} 1000,00$ & $2,9 \%$ \\
\hline $\mathrm{R} \$ 1001,00$ a 1600,00 & $13,5 \%$ \\
\hline $\mathrm{R} \$ 1601,00$ a 2000,00 & $16,3 \%$ \\
\hline $\mathrm{R} \$ 2001,00 \mathrm{a} 3000,00$ & $21,2 \%$ \\
\hline $\mathrm{R} \$ 3001,00 \mathrm{a} 4000,00$ & $16,3 \%$ \\
\hline Acima de $\mathrm{R} \$ 4000,00$ & $26,9 \%$ \\
\hline
\end{tabular}

No que tange aos níveis de escolaridade dos cônjuges, o ensino médio foi completado por $32,7 \%$ das mulheres, sendo esse o grau de escolaridade que predominou entre as respondentes. $O$ ensino superior foi concluído por $20,2 \%$ delas e a pós-graduação por $12,5 \%$. Assim como as mulheres, o grau de escolaridade que predominou entre os homens foi o ensino médio completo (27,9\%). O ensino superior foi concluído por $15,4 \%$ dos participantes masculinos, ao passo que $7,7 \%$ deles possuíam pós-graduação. Em termos de anos concluídos, a média de escolaridade feminina foi $12,34(D P=3,97)$ e masculina foi $11,40(D P=4,01)$.

Identificou-se que as mulheres apresentaram significativamente maior escolaridade em termos de anos concluídos $(U=3990,50$; $Z=2,07 ; p<0,05)$ e, também, nos níveis médio, superior e pósgraduação, em comparação aos homens $\left(X^{2}=154,24 ; p<0,01\right.$, $\mathrm{C}=0,77 ; \mathrm{p}<0,01)$. Apesar de a escolaridade feminina ser maior que a escolaridade masculina, a remuneração mensal média auferida pelas mulheres - $\mathrm{R} \$ 1.341,49 \quad(D P=1.276,70)$, foi menor que a remuneração mensal média recebida pelos homens - $\mathrm{R} \$ 2.112,45$ $(D P=1.543,89)$, sendo essa diferença estatisticamente significativa 
$(U=2.505,00 ; Z=5,28 ; p<0,01)$. Desse modo, constatou-se que a renda mensal masculina foi aproximadamente $57 \%$ maior que a renda mensal feminina.

No que diz respeito à participação no mercado de trabalho, constatou-se que $16,7 \%$ das mulheres não possuíam jornada de trabalho fora de casa, sendo classificadas como trabalhadoras do lar ou desempregadas, em contraposição a 5,9\% dos homens. Ademais, identificou-se ainda que os homens possuíam maior jornada de trabalho formal (fora do lar), em termos de carga horária semanal, do que as mulheres $\left(x^{2}=95,22 ; p<0,01, C=0,70 ; p<0,01\right)$, o que pode contribuir para a maior remuneração mensal masculina.

\subsection{Caracterização do relacionamento conjugal dos participantes}

A dimensão que obteve os mais altos escores foi a harmonia conjugal, tanto para homens $(M=5,05 ; D P=0,55)$, quanto para mulheres $(M=5,09 ; D P=0,55)$. Sendo assim, em média, os cônjuges indicaram que estão felizes com a relação de casal, que costumam agradar e satisfazer o companheiro, sendo gentis e praticando concessões em casos de conflitos. A dimensão evitação recebeu baixos escores médios de acordo com as respostas masculinas $(M=2,85 ; D P=0,71)$ e femininas $(M=2,81 ; D P=0,76)$. Desse modo, os participantes apontaram discordar total ou parcialmente no que diz respeito a valer-se de retração no intento de evitar desavenças e conversas que exijam a exposição de sentimentos e fraquezas pessoais.

Em reciprocidade negativa, também se obteve baixos escores médios nas respostas de homens $(M=2,61 ; D P=0,73)$ e de mulheres $(\mathrm{M}=2,90 ; D P=0,90)$. De tal maneira, os cônjuges discordaram total ou parcialmente sobre sua relação de casal ser permeada por brigas caracterizadas por aumento crescente de ofensas e xingamentos, bem como de sensação de iniquidade no relacionamento. Contudo, essa foi a única das três dimensões avaliadas da relação conjugal que apresentou diferença estatisticamente significativa entre as respostas de homens e de mulheres $(U=4431,500 ; Z=2,15 ; p<0,05)$. De tal forma, a mulher tende a indicar, de modo mais importante que 0 homem, que costuma fazer concessões e favores ao companheiro (tendo a impressão de que isso não é recíproco), considerando a relação conjugal desigual e caracterizada por interações conflitivas marcadas por cobranças e acusações.

\subsection{Variáveis sociodemográficas e relacionamento conjugal dos participantes}


A Tabela 2 apresenta os resultados com significância estatística no que diz respeito às relações entre as dimensões do relacionamento conjugal e as variáveis sociodemográficas dos participantes.

\begin{tabular}{lcc}
\hline Tabela 2 & & \\
Correlações entre o relacionamento conjugal e as variáveis sociodemográficas \\
\hline Relacionamento Conjugal & Variáveis & Coeficiente de correlação \\
\hline Reciprocidade negativa & sociodemográficas & $-0,21 *$ \\
masculina & Rendimento masculino & $-0,20 *$ \\
Evitação masculina & Escolaridade feminina & $-0,40 * *$ \\
& Escolaridade masculina & $-0,44 * *$ \\
& Rendimento feminino & $-0,20 *$ \\
& Rendimento masculino & $-0,34 * *$ \\
Harmonia feminina & Renda familiar total & $-0,39 * *$ \\
Reciprocidade negativa & Idade feminina & $-0,27 * *$ \\
feminina & Idade masculina & $-0,23 *$ \\
& No de pessoas na casa & $0,29 * *$ \\
& Escolaridade feminina & $-0,28^{* *}$ \\
& Escolaridade masculina & $-0,32 * *$ \\
& Rendimento feminino & $-0,22 *$ \\
& Rendimento masculino & $-0,38^{* *}$ \\
& Renda familiar total & $-0,36 * *$ \\
& No de pessoas na casa & $0,23 *$ \\
& Escolaridade feminina & $-0,28 * *$ \\
& Escolaridade masculina & $-0,29 * *$ \\
& Rendimento masculino & $-0,34 * *$ \\
& Renda familiar total & $-0,32 * *$ \\
\hline
\end{tabular}

Nota. Tabela elaborada pelos autores, com base nos resultados do estudo.

${ }^{*} \mathrm{p}<0,05{ }^{* *} \mathrm{p}<0,01$.

Mediante a Tabela 2, é possível identificar que as variáveis ligadas à escolaridade e aos rendimentos (tanto dos cônjuges quanto à renda familiar total) estiveram negativamente relacionadas à reciprocidade negativa feminina e à evitação, tanto para o homem quanto para a mulher. Os proventos do homem e a receita familiar total também se correlacionaram negativamente à reciprocidade negativa masculina, ou seja, quanto menor a renda e a escolaridade, mais ambos os cônjuges referiram que a relação de casal se caracteriza por reciprocidade negativa e por evitação.

A quantidade de pessoas residentes no domicílio familiar também se apresentou positivamente relacionada à emergência de brigas, discussões, troca de acusações e sentimentos de desigualdade relatados pela mulher, bem como à retração feminina com o intento de evitar conflitos conjugais. Destarte, quanto mais numerosa a 
família, maior a reciprocidade negativa e a evitação para as mulheres.

Constatou-se que a harmonia conjugal relatada pelas mulheres se correlacionou negativamente às idades masculina e feminina. Assim, quanto maior a idade dos membros do casal, menos harmoniosa a mulher considerava a sua relação conjugal. Vale ressaltar que a idade do homem esteve significativamente relacionada ao maior número de pessoas residentes no domicílio familiar $(\rho=0,24 ; \mathrm{p}<0,05)$, bem como à idade da mulher $(\rho=0,24 ; \mathrm{p}<0,05)$, e, consequentemente, ao maior número de filhos do casal, uma vez que em $95,1 \%$ dos casos residiam no domicílio unicamente cônjuges e filho(s), conforme já indicado anteriormente. Sendo assim, supõe-se que quanto maior a idade dos cônjuges, mais filhos tende a ter o casal, acarretando em um aumento da carga de trabalho e do estresse da mulher, o que contribui para a diminuição da harmonia conjugal por ela referida. É importante destacar que o tempo de união não esteve significativamente relacionado às dimensões avaliadas do relacionamento de casal.

\section{Discussão e conclusões}

O objetivo do presente estudo foi descrever a relação entre relacionamento conjugal e características sociodemográficas em arranjos familiares heteroafetivos. A hipótese inicial era de que variáveis tais como escolaridade, renda, número de pessoas que reside no domicílio familiar, idade dos cônjuges e tempo de união conjugal poderiam interferir na satisfação e na qualidade conjugal, abarcadas pela dimensão da harmonia conjugal, e/ou propiciar interações conflitivas destrutivas, marcadas por comportamentos de reciprocidade negativa e evitação.

A relação de casal dos participantes se caracterizou pela harmonia conjugal, sendo que os membros da díade discordam (total ou parcialmente) de se utilizarem de atitudes que envolvam reciprocidade negativa ou evitação para resolverem suas desavenças. No que diz respeito a tais considerações, vale ressaltar que uma relação de casal caracterizada como harmônica não se refere, necessariamente, a uma relação sem conflitos. Isso porque o conflito é inerente a todos os relacionamentos humanos, ou seja, é um fenômeno característico da vida e que pode resultar em novas oportunidades e transformações aos envolvidos (Bolze et al., 2013). Por conseguinte, há de se prever que os cônjuges, mesmo em relacionamentos considerados felizes e harmônicos, enfrentarão conflitos conjugais (Bolze et al., 2013; Narciso \& Ribeiro, 2009). Nesse sentido, faz-se necessário pontuar que o melhor preditor do 
sucesso do casamento em longo prazo é o modo como a díade conjugal lida com os seus conflitos (Narciso \& Ribeiro, 2009).

De tal forma, os baixos escores médios nos itens que versam sobre brigas, agressões, acusações e sentimentos de desigualdade (reciprocidade negativa), bem como sobre comportamentos de retração com o intento de evitar desavenças, exposição de sentimentos e fraquezas pessoais (evitação), indicam que os casais referem adotar pouco frequentemente estratégias destrutivas de resolução de conflitos. Assim, casais harmônicos parecem criar um clima de concordância na interação, incluindo mais positividade durante o conflito, mais aprovação e menos discordância e crítica (Madhyastha, Hamaker, \& Gottman, 2011). Tais resultados, em associação aos altos escores médios nos itens atinentes à percepção de felicidade e de satisfação na relação, exposição verbal de sentimentos e realização de concessões (harmonia conjugal), podem ser considerados importantes recursos dos participantes, haja vista que a boa qualidade da conjugalidade prediz melhores níveis de saúde nos cônjuges longitudinalmente, sendo compreendida, portanto, como fator de proteção ao desenvolvimento dos membros do casal e da família (Narciso \& Ribeiro, 2009; Wood, Crane, \& Keller, 2011).

Cabe ressaltar que apesar de os cônjuges concordarem com relação à harmonia conjugal e à evitação, verificou-se diferença estatisticamente significativa no que diz respeito à reciprocidade negativa: a mulher considera, mais predominantemente que o homem, a sua relação de casal desigual e permeada por interações conflitivas com troca de cobranças e de acusações. Esses resultados confirmam os achados de Perlin e Diniz (2005), que assinalam que mesmo em casais de dupla carreira que afirmam vivenciar casamentos felizes, as mulheres se consideram menos satisfeitas na relação conjugal em comparação aos homens. Conforme já discutido anteriormente, hipotetiza-se que um dos elementos associados a tal entendimento diz respeito ao fato de as mulheres contemporâneas, de modo geral, acumularem a jornada de trabalho fora do lar e as atividades domésticas, fato que pode fazer emergir um sentimento de iniquidade na relação.

De acordo com o esperado, as características da relação conjugal variaram em função das características sociodemográficas da amostra. Em consonância com a literatura pesquisada, identificou-se que a menor escolaridade e a menor renda dos cônjuges se relacionaram à maior prevalência de interações conjugais conflitivas permeadas por reciprocidade negativa e comportamentos evitativos. Esses dados corroboram a revisão de literatura que aponta associações entre baixo nível socioeconômico familiar e aumento das situações de conflito e estresse conjugal, bem como diminuição da qualidade conjugal (Dakin \& Wampler, 2008). Além disso, o grau de 
instrução formal dos cônjuges é considerado como uma das múltiplas variáveis que interferem na satisfação com o relacionamento de casal (Conger et al., 2010; Norgren et al., 2004; Rauer et al., 2008). Desse modo, um menor nível de escolaridade associa-se negativamente à percepção positiva da vida de casal (Conger et al., 2010; Dakin \& Wampler, 2008).

A quantidade de pessoas que reside no domicílio familiar também se apresentou positivamente relacionada à emergência de brigas, discussões, troca de acusações e sentimentos de desigualdade relatados pela mulher, bem como à retração feminina com o intento de evitar conflitos conjugais. Destarte, quanto mais numerosa a família, maior a reciprocidade negativa e a evitação para as mulheres. Destaca-se que Wendorf et al. (2011), com o objetivo de examinar o impacto do número de filhos na satisfação conjugal em diferentes culturas, constataram, em três países investigados, que o número de crianças tem um impacto negativo sobre a qualidade da relação conjugal. Os achados de Wendorf et al. (2011), no tocante à correlação negativa entre qualidade da conjugalidade e quantidade de filhos, são condizentes aos obtidos no presente estudo, ao se considerar que $95,1 \%$ dos casais entrevistados indicaram que 0 número de pessoas que vive na residência familiar corresponde a pai, mãe e filho(a)(s), ou seja, com ausência de outros parentes adultos ou agregados. Ressalta-se que, como a maioria dos participantes desta pesquisa residia, exclusivamente, junto ao cônjuge e filho(a)(s), pode-se pressupor que o maior número de filhos contribui para o maior estresse entre os cônjuges.

Constatou-se que a harmonia conjugal relatada pelas mulheres se correlacionou negativamente às idades masculina e feminina. Assim, quanto maior a idade dos membros do casal, menos harmoniosa a mulher considera a sua relação conjugal. Esse fenômeno pode ser parcialmente explicado pela pesquisa de Wendorf et al. (2011), a qual mostrou que para alguns casais a satisfação conjugal declina com o aumento da idade dos homens e das mulheres e pelo maior número de filhos, uma vez que o número de crianças é altamente correlacionado à idade dos cônjuges.

Em linhas gerais, verificou-se também que as mulheres parecem mais sensíveis às relações entre as variáveis sociodemográficas e a conjugalidade do que os homens. Além das variáveis relacionadas à escolaridade, renda, idade e carga horária de trabalho, considera-se que esse fenômeno pode ocorrer pelo fato de as mulheres, em geral, acumularem várias funções, pois mesmo assumindo participação no mercado de trabalho e no sustento familiar, elas ainda são identificadas como as principais responsáveis pelo cuidado dos filhos e pelas tarefas domésticas (Braz, Dessen, \& Silva, 2005; Jablonski, 2010; Wagner, Predebon, Mosmann, \& Verza, 2005). Dessa forma, a 
distribuição desigual das tarefas domésticas e de criação dos filhos pode concorrer para a insatisfação conjugal feminina.

Ressalta-se, ainda, a seguinte consideração: relatos dos respondentes sobre o efeito terapêutico gerado por participar da pesquisa. Alguns participantes indicaram que foi possível refletir sobre a relação conjugal após a aplicação do instrumento, informando aos pesquisadores que pretendiam conversar com o cônjuge sobre questões suscitadas por intermédio dos itens respondidos. Em determinados casos, houve a afirmação de que o questionário serviu de estímulo para abordar aspectos referentes ao relacionamento de casal que ainda não haviam sido discutidos, ou mesmo aqueles considerados tabus pelos cônjuges, o que leva a crer que a participação na pesquisa contribui para que os casais pensem a respeito das relações familiares estabelecidas. Destarte, enfatiza-se a importância de se fomentar espaços que permitam reflexões sobre a relação de casal. Salienta-se, assim, a importância da abordagem ao casal - e não apenas ao indivíduo - em programas e serviços de atenção à saúde.

Ademais, considerando que interações conflitivas ocorrem, em menor ou maior grau, em todas as díades conjugais, é premente pensar em ações, programas ou políticas públicas que oportunizem auxílio a casais que eventualmente passam por situação de desentendimento ou discórdia e, consequentemente, promovam o bem-estar familiar. Salienta-se a importância de que os profissionais de saúde atuem junto a casais no sentido de favorecer a utilização de estratégias saudáveis/construtivas de resolução de conflitos, incluindo validação dos pontos de vista do cônjuge e habilidades de comunicação (ou seja, relacionadas à harmonia conjugal), em detrimento às estratégias destrutivas, caracterizadas por atitudes defensivas, desprezo, cobrança, raiva ou afastamento (isto é, aquelas vinculadas à reciprocidade negativa e à evitação).

Do mesmo modo, ainda no tocante aos desdobramentos para a prática, concebe-se também a importância de políticas públicas voltadas à diminuição de iniquidades sociais e econômicas vivenciadas pelos casais e famílias, ponderando que essas se constituem em fator potencial de risco a trajetórias desenvolvimentais (Mills et al., 2012). Assim, faz-se a ressalva de que este estudo não tem a intenção de rotular ou caracterizar casais menos favorecidos economicamente como apresentando menor competência para estabelecer relações conjugais satisfatórias, mas sim, pontuar que a situação de vulnerabilidade social que os acomete deve ser levada em consideração na análise da conjugalidade.

Considera-se que a presente produção contribui com a literatura, por estabelecer discussões sobre aspectos concernentes ao relacionamento conjugal e a variáveis sociodemográficas, somandose ao corpo de conhecimentos já disponível sobre a temática. Não 
obstante, apresenta também limitações, sobretudo em face da adoção, para avaliação da conjugalidade, de um questionário ainda a ser validado, cujas respostas estavam dispostas em uma escala de pontos (isto é, questões com respostas fechadas), o que não permite aprofundar questões relativas à dinâmica relacional do casal. Entretanto, tal condição não inviabilizou ou mesmo invalidou os resultados do estudo, os quais acompanharam tendências previstas na literatura sobre a temática. Salienta-se, ainda, que é preciso ter cautela com os resultados apresentados por esta pesquisa, pois como o delineamento do estudo foi correlacional, não se podem inferir relações causais. Além disso, a maioria das correlações foi fraca ou moderada.

Finalmente, sugere-se que pesquisas futuras investiguem mais aprofundadamente relações entre a conjugalidade e os aspectos sociais e demográficos ora apresentados, recomendando-se a realização de estudos junto a populações com outras características (por exemplo, famílias monoparentais e casais homoafetivos), a fim de que se avalie se as tendências obtidas nessa pesquisa são mantidas, bem como a inclusão de outras medidas de comportamentos de comunicação, afeto e resolução de problemas que são bem referenciados na literatura sobre conjugalidade.

\section{Referências}

Askari, M., Noah, S. B. M., Hassan, S. A. B., \& Baba, M. B. (2012). Comparison the effects of communication and conflict resolution skills training on marital satisfaction. International Journal of Psychological Studies, 4(1), 182-195. doi: 10.5539/ijps.v4n1p182

Bigras, M. (2010). Développement du questionnaire FLOREAL. Unpublished manuscript. Université du Québec à Montréal, Montréal, Canada.

Birditt, K. S., Brown, E., Orbuch, T. L., \& McIlvane, J. M. (2010). Marital conflict behaviors and implications for divorce over 16 years. Journal of Marriage and Family, 72(5), 1188-1204. doi: 10.1111/j.1741-3737.2010.00758.x

Bolze, S. D. A., Schmidt, B., Crepaldi, M. A., \& Vieira, M. L. (2013). Relacionamento conjugal e táticas de resolução de conflito entre casais. Actualidades en Psicología, 27(114), 71-85.

Braz, M. P. Dessen, M. A., \& Silva, N. L. P. (2005). Relações conjugais e parentais: Uma comparação entre famílias de classes sociais baixa e média. Psicologia: Reflexão e Crítica, 18(2), 151-161. doi: 10.1590/S0102-79722005000200002 
Carter, B., \& McGoldrick, M. (1995). As mudanças no ciclo de vida familiar: Uma estrutura para a terapia familiar. Porto Alegre: Artes Médicas.

Conger, R. D., Conger, K. J., \& Martin, M. J. (2010). Socioeconomic status, family processes, and individual development. Journal of Marriage and Family, 72, 685-704. doi: 10.1111/j.17413737.2010.00725.x

Coutinho, S. M. S., \& Menandro, P. R. M. (2010). Relações conjugais e familiares na perspectiva de mulheres de duas gerações: "Que seja terno enquanto dure". Psicologia Clínica, 22(2), 83106. doi: 10.1590/S0103-56652010000200007

Dakin, J., \& Wampler, R. (2008). Money doesn't buy happiness, but it helps: Marital satisfaction, psychological distress, and demographic differences between low-and middle-income clinic couples. The American Journal of Family Therapy, 36, 300-311. doi: $10.1080 / 01926180701647512$

Féres-Carneiro, T. (1998). Casamento contemporâneo: o difícil convívio da individualidade com a conjugalidade. Psicologia: Reflexão e Crítica, 11(2), 379-394. doi: 10.1590/S010279721998000200014

Féres-Carneiro, T., \& Diniz Neto, O. (2010). Construção e dissolução da conjugalidade: Padrões relacionais. Paidéia, 20(46), 269278.

Féres-Carneiro, T., Ponciano, E. L. T., \& Magalhães, A. S. (2007). Família e casal: Da tradição à modernidade. In C. M. O. Cerveny (Ed.), Família em movimento (pp. 23-36). São Paulo, SP: Casa do Psicólogo.

Heckler, V. I., \& Mosmann, C. (2014). Casais de dupla carreira nos anos iniciais do casamento: Compreendendo a formação do casal, papéis, trabalho e projetos de vida. Barbarói, 41, 119147.

Jablonski, B. (2010). A divisão de tarefas domésticas entre homens e mulheres no cotidiano do casamento. Psicologia Ciência e Profissão, 30(2), 262-275. doi: 10.1590/S141498932010000200004

Madhyastha, T. M., Hamaker, E. L., \& Gottman, J. M. (2011). Investigating spousal influence using moment-to-moment affect data from marital conflict. Journal of Family Psychology, 25(2), 292-300.

Mills, R. S. L., Hastings, P. D., Helm, J., Serbin, L. A., Etezadi, J., Stack, D. M., Schwartzman, A. E., \& Li, H. H. (2012). Temperamental, parental, and contextual contributors to earlyemerging internalizing problems: A new integrative analysis approach. Social Development, 21(2), 229-253. doi: 10.1111/j.1467-9507.2011.00629.x 
Narciso, I., \& Ribeiro, M. T. (2009). Olhares sobre a conjugalidade. Lisboa: Coisas de Ler.

Norgren, M. B. P., Souza, R. M., Kaslow, F., Hammerschmidt, H., \& Sharlin, S. A. (2004). Satisfação conjugal em casamentos de longa duração: Uma construção possível. Estudos de Psicologia, 9(3), 575-584. doi: 10.1590/S1413-294X2004000300020

Oliveira, M. G. S. de, Falcone, E. M. de O., \& Ribas Jr, R. de C. (2009). A avaliação das relações entre a empatia e a satisfação conjugal: Um estudo preliminar. Interação em Psicologia, 13(2), 287-298.

Perlin, G. D. B. (2006). Casamentos contemporâneos: Um estudo sobre os impactos da interação família-trabalho na satisfação conjugal (Tese de Doutorado). Recuperado 26, março, 2015, de:

http://repositorio.unb.br/bitstream/10482/9274/1/2006_Giova naDalBiancoPerlin.pdf

Rauer, A. J., Karney, B. R., Garvan, C. W., \& Hou, W. (2008). Relationship risks in context: A cumulative risk approach to understanding relationship satisfaction. Journal of Marriage and Family, 70(2), 1122-1135. doi: 10.1111/j.17413737.2008.00554.x

Rolim, K. I, \& Wendling, M. I. (2013). A história de nós dois: Reflexões acerca da formação e dissolução da conjugalidade. Psicologia Clínica, 25(11), 165-180.

Silvares, E. F. M., \& Souza, C. L. (2008). Discórdia conjugal: Distúrbios psicológicos infantis e avaliação diagnóstica comportamental-cognitiva. Psicologia: Teoria e Prática, 10(1), 200-213.

Scorsolini-Comin, F., \& Santos, M. A. (2011a). Relações entre bemestar subjetivo e satisfação conjugal na abordagem da psicologia positiva. Psicologia: Reflexão e Crítica, 24(4), 658665. doi: 10.1590/S0102-79722011000400005

Scorsolini-Comin, F., \& Santos, M. A. (2011b). Laços de família: A conjugalidade como dimensão de análise do desenvolvimento. Polêm!ca, 10(2), 288-298.

Vieira, M. L., Lacerda, A. L. R., Vieira, M. C., \& Seidl-de-Moura, M. L. (2011). Características sociodemográficas e aspectos psicossociais de famílias brasileiras residentes em capitais e cidades pequenas do interior. Barbarói, 34(1), 60-79.

Wagner, A., \& Falcke, D. (2001). Satisfação conjugal e transgeracionalidade. Psicologia Clínica, 13(2), 11-24.

Wagner, A., Predebon, J., Mosmann, C., \& Verza, F. (2005). Compartilhar Tarefas? Papéis e funções de pai e mãe na família contemporânea. Psicologia: Teoria e Pesquisa, 21(2), 181-186.

Wendorf, C. A., Lucas, T., Imamoğlu, E. O., Weisfeld, C. C., \& Weisfeld, G. E. (2011). Marital satisfaction across three 
cultures: Does the number of children have an impact after accounting for other marital demographics? Journal of CrossCultural Psychology, 42(3), 340-354. doi: $10.1177 / 0022022110362637$

Whitaker, C. A. (1995). As funções do casal. In M. Andolfi, C. Angelo, \& C. Saccu (Eds.), O casal em crise (pp. 21-28). São Paulo, SP: Summus.

Willi, J. (1995). A construção diádica da realidade. In M. Andolfi, C. Angelo, \& C. Saccu (Eds.), O casal em crise (pp. 38-46). São Paulo, SP: Summus.

Wood, N. D., Crane, D. R., \& Keller, P. S. (2011). Tracking marital adjustment, hostility, and physical functioning across time in a therapy population: A biopsychosocial model. Contemporary Family Therapy, 33(3), 242-252. doi: 10.1007/s10591-0119164-4.

Zordan, E. P., Wagner, A., \& Mosmann, C. (2012). O perfil de casais que vivenciam divórcios consensuais e litigiosos: Uma análise das demandas judiciais. Psico-USF, 17(2), 185-194.

\section{Endereço para correspondência \\ Beatriz Schmidt}

Universidade Federal do Rio Grande do Sul

Instituto de Psicologia

Núcleo de Infância e Família - NUDIF

Rua Ramiro Barcelos, 2600, sala 111, Santa Cecília, CEP 90035-003, Porto Alegre RS, Brasil

Endereço eletrônico: psi.beatriz@gmail.com

\section{Simone Dill Azeredo Bolze}

Universidade Federal de Santa Catarina

Departamento de Psicologia

Laboratório de Pesquisa em Psicologia da Saúde, Família e Comunidade - LABSFAC

Campus Universitário, Trindade, CEP 88040-970, Florianópolis - SC, Brasil

Endereço eletrônico: simoneazeredo@yahoo.com.br

\section{Mauro Luis Vieira}

Universidade Federal de Santa Catarina

Departamento de Psicologia

Núcleo de Estudos e Pesquisas em Desenvolvimento Infantil - NEPeDI

Campus Universitário, Trindade, CEP 88040-970, Florianópolis - SC, Brasil

Endereço eletrônico: maurolvieira@gmail.com

\section{Maria Aparecida Crepaldi}

Universidade Federal de Santa Catarina

Departamento de Psicologia

Laboratório de Pesquisa em Psicologia da Saúde, Família e Comunidade - LABSFAC

Campus Universitário, Trindade, CEP 88040-970, Florianópolis - SC, Brasil

Endereço eletrônico: maria.crepaldi@gmail.com

Recebido em: 09/06/2014

Reformulado em: 01/04/2015

Aceito para publicação em: 08/06/2015

\section{Notas}


* Doutoranda em Psicologia pela Universidade Federal do Rio Grande do Sul; Psicóloga; Especialista em Saúde da Família; Mestre em Psicologia.

** Doutoranda em Psicologia pela Universidade Federal de Santa Catarina; Psicóloga; Especialista em Psicologia Clínica - Terapia Relacional Sistêmica; Mestre em Psicologia.

*** Professor do Departamento de Psicologia e do Programa de Pós-Graduação em Psicologia da Universidade Federal de Santa Catarina; Psicólogo; Mestre em Psicologia Experimental; Doutor em Psicologia Experimental.

**** Professora do Departamento de Psicologia e do Programa de Pós-Graduação em Psicologia da Universidade Federal de Santa Catarina; Psicóloga; Mestre em Psicologia Clínica; Doutora em Saúde Mental.

\section{Agradecimentos}

À Coordenação de Aperfeiçoamento de Pessoal de Nível Superior (CAPES), pela concessão de bolsa de pós-graduação à primeira e à segunda autora;

Às famílias participantes e aos pesquisadores vinculados ao projeto " $A$ transmissão intergeracional da violência: a relação do conflito conjugal e parental com a agressividade entre pares de crianças de quatro a seis anos";

À Regina Campos Lima Sartori, pelas sugestões de aprimoramento à discussão dos dados sociodemográficos. 